\title{
SUPERDOTAÇÃO/ALTAS HABILIDADES PERCEBIDAS POR PAIS E PROFESSORES DO ENSINO FUNDAMENTAL, NÍVEL I
}

\author{
SUPERDOTACIÓN/ALTAS CAPACIDADES PERCIBIDAS POR PADRES Y \\ DOCENTES DE ENSEÑANZA BÁSICA, $1^{\circ}$ GRADO DE PRIMARIA
}

\author{
GIFTED/HIGH ABILITIES PERCEIVED BY PARENTS AND TEACHERS OF \\ ELEMENTARY SCHOOL, LEVEL I
}

\author{
Alexandra Imaculada de Oliveira e MEDEIROS ${ }^{1}$ \\ Letycia Pedroso ALENCAR ${ }^{2}$ \\ Nathalya Elizabeth Ferrer UBEDA ${ }^{3}$ \\ Betânia Jacob Stange LOPES ${ }^{4}$
}

RESUMO: As políticas educacionais de inclusão propõem medidas que garantem a participação e desenvolvimento de estudantes com superdotação/altas habilidades, todavia, há um grande desafio no que tange à sua identificação. A realização deste estudo objetivou identificar a percepção de pais e professores quanto às características de superdotação/altas habilidades de seus filhos e alunos. O estudo foi realizado no Ensino Fundamental, Nível I, de uma cidade de pequeno porte no estado de São Paulo. Utilizou-se questionários para pais e professores a fim de realizar coleta de informações. A análise dos dados revelou que os pais demonstraram grandes expectativas quanto ao potencial de seus filhos, enquanto que os professores foram mais ponderados ao escolher os indicadores referentes ao potencial de seus alunos. Ambos apresentaram conhecimento superficial quanto ao assunto e os professores fizeram poucas indicações no item Criatividade, elemento indispensável no processo, pois envolve originalidade de pensamento na solução de problemas.

PALAVRAS-CHAVE: Superdotação. Ensino fundamental. Indicadores.

RESUMEN: Las políticas educativas de inclusión proponen medidas que garantizan la participación y el desarrollo de estudiantes con sobredotación/altas capacidades, sin embargo existe un gran desafio en lo que se refiere a su identificación. La realización de esta investigación tuvo como objetivo identificar la percepción de los padres y docentes en relación con las características en sus hijos y estudiantes con superdotación/altas capacidades. La investigación fue realizada con estudiantes del nível Básico, $1^{\circ}$ grado de primaria de una ciudad pequeña del estado de San Paulo. Se utilizaron cuestionarios para padres y professores con la finalidad de obtener

${ }^{1}$ Centro Universitário Adventista de São Paulo (UNASP), Engenheiro Coelho - SP - Brasil. Graduação em Pedagogia. E-mail: Alexandra.imaculada@gmail.com.

${ }^{2}$ Centro Universitário Adventista de São Paulo (UNASP), Engenheiro Coelho - SP - Brasil. Graduação em Pedagogia. E-mail: Letycia.alencar@hotmail.com.

${ }^{3}$ Centro Universitário Adventista de São Paulo (UNASP), Engenheiro Coelho - SP - Brasil. Graduação em Pedagogia. E-mail: Nathalya.ubeda@ hotmail.com..

${ }^{4}$ Centro Universitário Adventista de São Paulo (UNASP), Engenheiro Coelho - SP - Brasil. Assessora do PROAP. E-mail: betania.stange@unasp.edu.br 
información. El análisis de los datos reveló que los padres demostraron grandes expectativas en relación con el potencial de sus hijos, mientras que los professores fueron más ponderados al elegir los indicadores referentes al potencial de los alumnos. Ambos manifestaron conociminetos superficiales con el assunto de la investigación, y los profesores hicieron pocas observaciones en relación al item: Creatividad, elemento indispensable en el processo, pues involucra originalidad de pensamiento en la solución del problema.

PALAVRAS-CLAVES: Superdotación. Enseñanza básica. Indicadores.

ABSTRACT: The inclusionary educational policies propose measures that guarantee the right of participation and development of gifted/highly rated students, however, there is a major challenge in their identification. Therefore, the accomplishment of this essay aims to identify the perception of parents and teachers regarding the characteristics of highly gifted/high abilities of their children and students. The research was performed with parents and teachers of Elementary School, Level I, of a small city in the Metropolitan Region of Campinas, state of São Paulo. The study was based on a questionnaire for parents and teachers to collect information. Analysis of the data revealed that parents expressed high expectations about their children's potential, teachers were more thoughtful when selecting the indicators for the potential of their students. Parents and teachers presented superficial knowledge on the subject. Still, there was a very low number of teachers that indicated Creativity, indispensable element in this process, because it involves originality of thought and ability to solve problems.

KEYWORDS: Gifted. Elementary school. Factors.

\section{Introdução}

O tema superdotação tem sido motivo de inúmeras pesquisas, porém percebe-se a existência de muitas dúvidas quanto à sua identificação. As características gerais da superdotação podem ser percebidas no indivíduo que possui diferentes talentos e habilidades, alcançando excelência em áreas distintas na educação ou no desenvolvimento infantil. Todavia, é preciso que exista uma constância em suas habilidades e ainda uma demonstração de alto desempenho na área da Superdotação (BRASIL, 2006).

As políticas educacionais e de inclusão, propõem medidas que garantam o direito de participação e desenvolvimento de alunos com Superdotação/Altas Habilidades (S/AH) na rede regular de ensino. De acordo com o artigo $5^{\circ}$ da Resolução CNE/CEB no 2, de setembro de 2001 (p.2), são público alvo da Educação Especial (PAEE) os que apresentarem: 
III - altas habilidades/superdotação, grande facilidade de aprendizagem que os leve a dominar rapidamente conceitos, procedimentos e atitudes. (BRASIL, 2001).

Segundo a PNEEPEI (2008), esse grupo de estudantes demonstram:

Potencial elevado em qualquer uma das seguintes áreas, isoladas ou combinadas: intelectual, acadêmica, liderança, psicomotricidade e artes. Também apresentam elevada criatividade, grande envolvimento na aprendizagem e realização de tarefas em áreas de seu interesse (BRASIL, 2008, p. 15).

Por serem sujeitos que possuem grande criatividade, envolvimento na tarefa e habilidades acima da média entre seus pares, são considerados importantes para a sociedade, uma vez que a modernidade trouxe a necessidade de sujeitos talentosos e criativos. Esses estudantes precisam ser assistidos desde os primeiros anos da infância, quando possuem condições mais favoráveis de desenvolvimento da sua criatividade e do seu talento. É responsabilidade da família e da escola a percepção dessa superdotação em seus filhos e alunos (MAIA-PINTO; FLEITH, 2002).

Apesar da importância de se desenvolver um trabalho especializado com essas crianças, os alunos com S/AH nem sempre são percebidos ou identificados. Um estudo feito pela Organização Mundial da Saúde (OMS) aponta a expectativa da presença de cerca de $5 \%$ dos alunos matriculados.

Pesquisa realizada por Kahl (2011) com profissionais de educação, mostra que $20 \%$ dos docentes sentem-se inseguros quanto ao tema S/AH e $80 \%$ apresentaram conhecimento razoável. Essa realidade se estende não somente ao contexto escolar, mas também à família.

Diante desse contexto, surgiu o seguinte questionamento: Qual a percepção de pais e professores quanto aos indicadores de superdotação/altas habilidades de seus filhos e alunos?

Para elucidação do questionamento proposto, estabeleceu-se por objetivo geral: identificar a percepção de pais e professores sobre as características de superdotação/altas habilidades em seus filhos e alunos. Para atingir essa meta, estabeleceram-se como objetivos específicos: mapear os indicadores de superdotação/altas habilidades apontados por pais e professores a partir da Teoria dos Três anéis de Renzulli, e comparar a percepção de pais e professores quanto à escolha de características de superdotação/altas habilidades dos alunos estudados. 


\section{Conhecendo a Superdotação/Altas Habilidades}

O assunto superdotação/altas habilidades (S/AH) não é novo, todavia a discussão e interesse pelo tema tem se acentuado na atualidade. Estudiosos como Guenther (2006), Fleith (2007) e Marques (2010), destacam a importância do tema e esclarecem que o desenvolvimento de pessoas com S/AH pode causar não somente avanço para o indivíduo, mas também para a sociedade, que precisa cada vez mais de profissionais talentosos e criativos. Com o potencial desenvolvido, o indivíduo tem melhor qualidade de vida e pode exercer a cidadania plenamente. Levando em consideração o mundo atual em constante mudança, Marques (2010), aponta o indivíduo talentoso como tendo fator diferencial competitivo por contribuir para a formação de um grupo social mais capacitado a solucionar problemas ao ler o mundo sob outro olhar.

Quando se fala de pessoas com S/AH, uma das dificuldades é a utilização adequada da nomenclatura, pela variedade de terminologias utilizadas de forma inadequada. Santos e Peripolli (2011) ratifica essa ideia ao explicitar que a nomenclatura superdotação gera confusão de conceitos, pela comparação à criança precoce, prodígio e ao gênio.

A criança precoce apresenta alguma habilidade desenvolvida prematuramente em qualquer área do conhecimento. Já a prodígio, executa tarefas em nível profissional, podendo ser comparado a um adulto. A genialidade deve ser atribuída a pessoas que fizeram grandes contribuições acima de seu tempo, como Gandhi, Einstein e Jobs, que levaram a sociedade a grandes revoluções (VIRGOLIM, 2007).

\section{Diferentes olhares sobre Superdotação/Altas Habilidades}

Por ser um tema polêmico, diferentes estudiosos se interessaram por estudar a superdotação/altas habilidades (S/AH) para verificar a avaliação da inteligência e identificar o potencial superior com ênfase em seu desenvolvimento. Tais pesquisadores tiveram diferentes visões sobre a superdotação, portanto não chegam a um consenso. Gagné (2009) associou a confusão de definições propostas com a Torre de Babel da Bíblia. Dentre as inúmeras teorias desenvolvidas para explicar o fenômeno, destaca-se a contribuição de Gardner (1994), Gagné (2000) e Renzulli (1986).

A teoria de Gardner (1994), também conhecida como a Teoria das Inteligências Múltiplas, defende que pessoas normais são capazes de resolver problemas em até oito diferentes áreas intelectuais relativamente dependentes em graus variados, a saber: 
linguística, lógico-matemática, cinestésica, musical, espacial, interpessoal, intrapessoal e naturalista. Em sua concepção, o superdotado tem maior precocidade no conteúdo em uma ou mais áreas intelectuais.

Gagné (2000), por sua vez, desenvolveu o Modelo Diferenciado de Superdotação e Talento. Segundo o pesquisador, a superdotação é uma herança genética e o indivíduo superdotado expressa suas habilidades de forma espontânea e natural, que também são chamadas de dons. Porém, o talento se distingue por ser o resultado da combinação de habilidades naturais e os contextos sociais em que o indivíduo se encontra, como os ambientes familiar e escolar. $\mathrm{O}$ autor denomina as áreas de domínio superior como: intelectual, criativa, sócioafetiva, sensório-motora e percepção extrassensorial.

Renzulli (1986), estudioso de superdotação, desenvolveu a teoria dos três anéis. Segundo essa concepção, a superdotação é resultado de três dimensões em interação: Habilidade Acima da Média, Envolvimento com a Tarefa e a Criatividade. A Figura 1 representa a interação existente entre essas dimensões.

Figura 1: Diagrama da Teoria dos Três Anéis de Renzulli

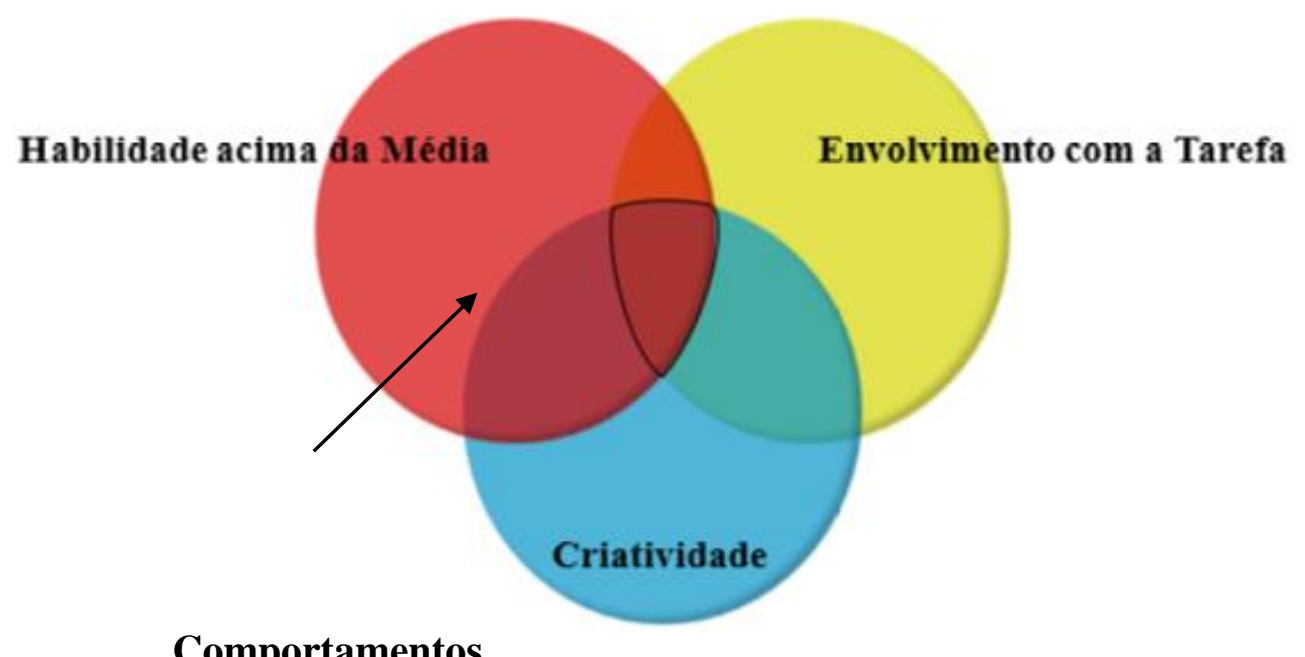

\section{Comportamentos}

Fonte: Marques (2010, p. 46) - Adaptado pelas autoras

$\mathrm{Na}$ proposta dos três anéis, cada círculo representa um elemento da superdotação: a 'Habilidade Acima da Média' é compreendida como "grande capacidade de processar informação, integrar experiências que resultam na adaptação de respostas a novas situações e capacidade de pensamento abstrato" (RENZULLI, 1986, p. 14); o 'Envolvimento com a Tarefa' é visto como sinônimo de motivação, 
perseverança e dedicação nas ações aplicadas nas áreas de interesse do indivíduo; e o terceiro anel é a 'Criatividade', que envolve a originalidade de pensamento e talento para solucionar problemas. $\mathrm{Na}$ intersecção dos três círculos ou anéis, encontra-se a superdotação.

De acordo com esse modelo, nem sempre o indivíduo desenvolverá todas as características igualmente, mas poderá aumentar seu potencial de acordo com as oportunidades recebidas. Virgolim (2007), destaca que teoria de Renzulli (1986) ampliou a identificação de alunos com S/AH pois leva em consideração qualquer área geral ou específica de desempenho.

A identificação de superdotados é um processo complexo, pois, segundo Renzulli (1986), a manifestação do fenômeno ocorre de forma ampla e multidimensional, com aspectos quantitativos e qualitativos. Devido a essa característica, é possível detectar a superdotação por meio de diferentes meios como formulários, entrevistas, entre outros. Tais instrumentos devem envolver tanto a pessoa que apresente as características de $\mathrm{S} / \mathrm{AH}$, quanto os indivíduos à sua volta como familiares e professores.

Através dos anos, os testes de inteligência deixaram de ser o núcleo de identificação principal e passaram a ser substituídos por ferramentas que envolvem maior interação e observação dos indicadores de S/AH. Marques (2010) aponta a percepção de indicadores como um "gatilho" inicial para a identificação de indivíduos superdotados.

\section{A relação entre pais e professores com Superdotação/Altas Habilidades}

O lar é a base sólida para a criança, pois dará suporte para o desenvolvimento desta desde os primeiros anos de vida até a fase adulta. Oliveira (2008) afirma que este grupo social (pais) atua diretamente no desenvolvimento da moral, personalidade e evolução escolar de seus filhos. Devido a isso, a família desempenha papel fundamental para a identificação das altas habilidades em seus filhos (RECH, 2007).

A partir da identificação de S/AH, Silva e Fleith (2008) observaram a mudança na dinâmica familiar, pois os pais buscam promover as habilidades dos filhos e suprir suas demandas e necessidades. Eles ainda demonstram confusão em relação ao futuro dos filhos, pois ficam incertos se isso seria benéfico ou prejudicial. É comum as pessoas do círculo familiar do indivíduo sentirem angústias e dúvidas com relação a mitos, 
informações errôneas e falta de conhecimento dos recursos disponíveis. Oliveira (2008) acrescenta que os familiares se sentem ansiosos e impotentes para lidar com um sujeito que possui essas características.

A partir de um estudo de caso, Rech (2007) observou grande esforço por parte dos pais para que sua filha, já identificada com altas habilidades, tivesse um suporte consistente. Ao encontrar grandes obstáculos diante da falta de estímulos oferecidos por parte da escola, foi percebida grande preocupação por parte da família por não saber como buscar mais ajuda. O conhecimento e observação dos pais foi fundamental para que a menina obtivesse o estímulo necessário para desenvolver suas habilidades.

Algo que também tem sido motivo de grande reflexão sobre esse tema é a percepção de S/AH no âmbito escolar. Pensando nisso, muitos estudiosos (CAMPBELL ; VERNA,1998; MAIA-PINTO; FLEITH, 2002; VIRGOLIM, 2007) têm destacado a importância da atuação da escola e do professor no processo de identificação do aluno. Todavia, estes muitas vezes não adotam medidas ou instrumentos para identificação, e nem os atendem de forma diferenciada.

Em sua pesquisa, Maia-Pinto e Fleith (2002), ressaltaram que muitos professores afirmam nunca ter trabalhado com alunos superdotados. Esses profissionais possuem um conhecimento superficial do conceito de S/AH, do processo de identificação e não receberam orientação específica sobre práticas educacionais compatíveis com as necessidades de alunos superdotados.

Por estar em contato com a criança diariamente, o professor pode perceber sinais de um potencial acima da média e, assim, encaminhar o aluno para investigação e, posteriormente, identificação. Portanto, as relações entre professor e aluno são de suma importância para o seu desenvolvimento. É nesse momento que a política adotada pela escola pode incentivar ou atrapalhar esse processo. Estratégias de ensino, cursos para especialização e o clima da sala de aula farão a diferença na educação dos alunos superdotados (MAIA-PINTO; FLEITH, 2002).

\section{Metodologia}

A abordagem que foi utilizada nesse estudo foi a quantitativa, a qual, segundo Fonseca (2002), traduz em números as opiniões e informações para serem classificadas e analisadas. Esse estudo teve a intenção de quantificar os resultados dos dados coletados por meio dos questionários realizados com os pais e professores e 
posteriormente analisá-los com a finalidade de compreender a relação das percepções dos participantes quanto às características de S/AH presentes em seus filhos e alunos.

O estudo foi caracterizado do tipo transversal, por "[...] se tratar de um corte instantâneo feito de uma população por meio de uma amostragem, como uma fotografia" (HOCKMAN et al, 2005). A coleta de dados aconteceu em um período de tempo breve e fixo, com a finalidade de reunir informações dos participantes no mesmo espaço de tempo.

\section{Local e participantes}

A pesquisa foi realizada em uma escola particular confessional que atende 436 alunos, em um município de pequeno porte da região metropolitana de Campinas, interior de São Paulo. Participaram deste estudo 6 professores e 160 pais de alunos matriculados no $3^{\circ}, 4^{\circ}$ e $5^{\circ}$ anos do Ensino Fundamental, Nível I. Os pais foram designados pelos códigos PP (participante pai), seguidos pelo número do ano, turno, número de chamada, (PP3101, PP3102...) e os professores, pelo código PF, também seguida de números sequenciais (PF01, PF02).

\section{Instrumentos}

Para a seleção/inclusão e caracterização dos pais, professores e coleta de dados foram empregados: (a) Ficha de Identificação de Pais/Professores - que teve como objetivo a obtenção de dados de identificação e informação de contato (nome, telefone, endereço eletrônico, entre outras); (b) Ficha de Caracterização de Pais/Professores com objetivo de registro de informações sobre os pais e professores como: idade, escolarização, renda e conceitos sobre o tema; (c) Formulário de Identificação de Indicadores de Superdotação/Altas Habilidades (MARQUES, 2010) com objetivo de levantar, dos pais e professores, os comportamentos considerados como possíveis indicadores de $\mathrm{S} / \mathrm{AH}$ de seus filhos e alunos.

\section{Coleta e análise de dados}

O procedimento adotado pelo grupo foi, primeiramente, o contato com gestor escolar propondo o estudo, esclarecimento dos procedimentos e aprovação da pesquisa. Após a autorização, os professores e pais foram o alvo da pesquisa. Aqueles que 
optaram por tomar parte do estudo receberam os formulários da pesquisa descritos acima para preenchimento conforme orientação das pesquisadoras.

As respostas dos pais e professores dos questionários foram organizadas em uma planilha que especificava em suas colunas os conjuntos de indicadores associados a cada uma das características representadas pela Teoria dos Três Anéis (RENZULLI, 1986). As informações classificadas de acordo com a planilha foram organizadas no Quadro I. Nele, constam seis colunas que apresentam três conjuntos de informações classificados de acordo com os indicadores e o respectivo anel a eles correspondente, conforme proposto por Marques (2010).

Quadro I: Indicadores* e traços de comportamento da Teoria dos Três Anéis**

\begin{tabular}{|c|c|c|c|c|c|}
\hline$*$ & $\begin{array}{l}\text { Habilidades } \\
\text { Acima da } \\
\text { Média*** }\end{array}$ & $*$ & Criatividade** & $*$ & Envolvimento com a Tarefa** \\
\hline 1 & $\begin{array}{l}\text { Aprende fácil e } \\
\text { rapidamente. }\end{array}$ & 2 & $\begin{array}{l}\text { Original, imaginativo, } \\
\text { criativo, não } \\
\text { convencional. }\end{array}$ & 3 & $\begin{array}{l}\text { Amplamente informado; informado } \\
\text { em áreas comuns. }\end{array}$ \\
\hline 6 & $\begin{array}{l}\text { Persuasivo, capaz } \\
\text { de influenciar os } \\
\text { outros. }\end{array}$ & 4 & $\begin{array}{l}\text { Pensa de forma } \\
\text { incomum para resolver } \\
\text { problemas. }\end{array}$ & 5 & $\begin{array}{l}\text { Persistente, independente, } \\
\text { autodirecionado (faz coisas sem } \\
\text { que seja mandado). }\end{array}$ \\
\hline 7 & $\begin{array}{l}\text { Mostra senso } \\
\text { comum; pode não } \\
\text { tolerar tolices. }\end{array}$ & 0 & $\begin{array}{l}\text { Esperto ao fazer coisas } \\
\text { com matérias comuns. }\end{array}$ & 8 & $\begin{array}{l}\text { Inquisitivo, cético, curioso sobre } \\
\text { como e por que das coisas. }\end{array}$ \\
\hline 2 & $\begin{array}{l}\text { Entende a } \\
\text { importância da } \\
\text { natureza (tempo, } \\
\text { lua, sol, estrelas, } \\
\text { solo, etc.) }\end{array}$ & 1 & $\begin{array}{l}\text { Habilidades nas artes } \\
\text { (música, dança, } \\
\text { desenho, etc.) }\end{array}$ & 9 & $\begin{array}{l}\text { Adapta-se a uma variedade de } \\
\text { situações e novos ambientes. }\end{array}$ \\
\hline 3 & $\begin{array}{l}\text { Vocabulário } \\
\text { excepcional, } \\
\text { verbalmente } \\
\text { fluente. }\end{array}$ & 7 & Flexível, aberto. & 5 & $\begin{array}{l}\text { Trabalhador independente, mostra } \\
\text { iniciativa. }\end{array}$ \\
\hline 4 & $\begin{array}{l}\text { Aprende } \\
\text { facilmente novas } \\
\text { línguas. }\end{array}$ & 9 & $\begin{array}{l}\text { Mostra insight e } \\
\text { percepções incomuns. }\end{array}$ & 8 & $\begin{array}{l}\text { Versátil, muitos interesses, além da } \\
\text { idade cronológica. }\end{array}$ \\
\hline 6 & $\begin{array}{l}\text { Bom julgamento, } \\
\text { lógico. }\end{array}$ & 2 & $\begin{array}{l}\text { Resiste à rotina e } \\
\text { repetição. }\end{array}$ & 0 & $\begin{array}{l}\text { Demonstra alto nível de } \\
\text { sensibilidade e empatia em relação } \\
\text { aos outros. }\end{array}$ \\
\hline
\end{tabular}




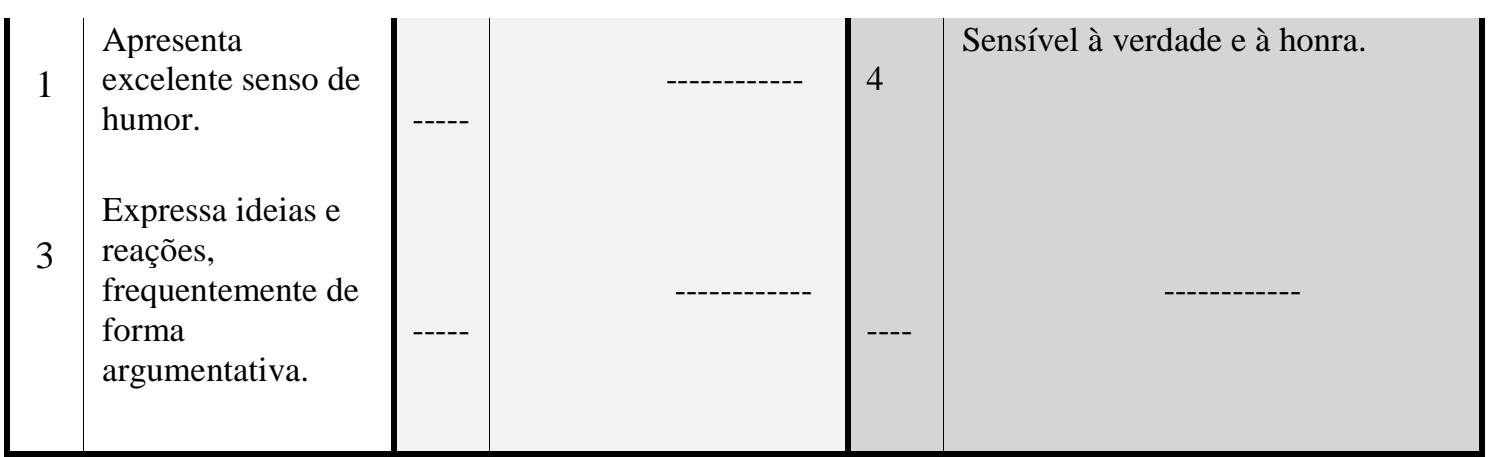

Fonte: Lopes (2016)

\section{Resultados}

Este estudo teve por objetivo identificar as características de S/AH na percepção de pais e professores quanto à capacidade de seus filhos e alunos, a partir da seleção dos indicadores de S/AH pelos pais e professores, para cada um dos anéis. Destacaram-se os resultados apresentados pelos pais que apresentaram o maior número de indicadores (três de cada turma, participaram da pesquisa doze alunos) nas três áreas apresentadas na Teoria dos Três Anéis, de Renzulli (1986), a saber: Habilidade Acima da Média; Criatividade e Envolvimento com a Tarefa, conforme Tabela 1.

Tabela 1: Percepção de pais quanto a presença de indicadores de Superdotação em seus filhos

\begin{tabular}{|c|c|c|c|c|c|c|c|c|c|c|c|c|c|c|c|c|c|c|c|c|c|c|c|c|c|}
\hline Código do aluno & $\mathrm{Q} 01$ & Q06 & $\mathrm{Q07}$ & Q12 & Q13 & Q14 & $\overline{Q 16}$ & Q21 & Q23 & Q17 & Q19 & $\bar{Q} 22$ & $\mathrm{Q} 04$ & $\bar{Q} 02$ & $\mathrm{Q10}$ & Q11 & Q18 & Q20 & $\bar{Q} 24$ & $\mathrm{Q} 15$ & $\overline{Q 03}$ & $\mathrm{Q05}$ & Q08 & Q09 & TOTAL \\
\hline A3101 & $Y$ & $\mathrm{Y}$ & $\mathrm{Y}$ & $\mathrm{Y}$ & $\mathrm{Y}$ & & & & & & & & & & $\mathrm{Y}$ & & & & $\mathrm{Y}$ & & & & & $\mathrm{Y}$ & 8 \\
\hline A3106 & $Y$ & $\mathrm{Y}$ & & $\mathrm{Y}$ & & $\mathrm{Y}$ & $Y$ & $\mathrm{Y}$ & $\bar{Y}$ & $Y$ & & $Y$ & & & $\bar{Y}$ & $\mathrm{Y}$ & & 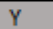 & $\bar{Y}$ & $\bar{Y}$ & & & $\bar{Y}$ & $\bar{Y}$ & 16 \\
\hline A3105 & $Y$ & $\mathrm{Y}$ & & $\mathrm{Y}$ & $\mathrm{Y}$ & $\mathrm{Y}$ & $\mathrm{Y}$ & $\mathrm{Y}$ & $\mathrm{Y}$ & $Y$ & $\mathrm{Y}$ & $\mathrm{Y}$ & $\mathrm{Y}$ & $\bar{Y}$ & $\mathrm{Y}$ & & $Y$ & $\mathrm{Y}$ & $\mathrm{Y}$ & $\mathrm{Y}$ & & & $\mathrm{Y}$ & $\mathrm{Y}$ & 20 \\
\hline A3222 & $\mathrm{Y}$ & & $\mathrm{Y}$ & $\mathrm{Y}$ & $\mathrm{Y}$ & $\mathrm{Y}$ & & & $\mathrm{Y}$ & $Y$ & & & $\mathrm{Y}$ & & & $\mathrm{Y}$ & & & $\mathrm{Y}$ & $\mathrm{Y}$ & $\mathrm{Y}$ & $\mathrm{Y}$ & $\mathrm{Y}$ & $\mathrm{Y}$ & 15 \\
\hline A3230 & $\mathrm{Y}$ & $\mathrm{Y}$ & $\mathrm{Y}$ & $\mathrm{Y}$ & $\mathrm{Y}$ & & $\mathrm{Y}$ & $\mathrm{Y}$ & $\mathrm{Y}$ & & $\mathrm{Y}$ & $\mathrm{Y}$ & $\mathrm{Y}$ & $\mathrm{Y}$ & & & $Y$ & & $\mathrm{Y}$ & & $\mathrm{Y}$ & $\mathrm{Y}$ & $\mathrm{Y}$ & $\mathrm{Y}$ & 18 \\
\hline A3202 & $Y$ & & $\mathrm{Y}$ & $\mathrm{Y}$ & & & $\mathrm{Y}$ & & & & & & & $\mathrm{Y}$ & $\mathrm{Y}$ & & $Y$ & & & & $\mathrm{Y}$ & & & & 8 \\
\hline A4101 & $Y$ & $\mathrm{Y}$ & & $\mathrm{Y}$ & & & $Y$ & & $\mathrm{Y}$ & $Y$ & & & & $Y$ & $Y$ & $\mathrm{Y}$ & & $\mathrm{Y}$ & $\mathrm{Y}$ & & & & $\mathrm{Y}$ & $\mathrm{Y}$ & 13 \\
\hline A4103 & $Y$ & $\mathrm{Y}$ & & $\mathrm{Y}$ & $\mathrm{Y}$ & $\mathrm{Y}$ & $\mathrm{Y}$ & $\mathrm{Y}$ & $\mathrm{Y}$ & $Y$ & & $Y$ & & $\mathrm{Y}$ & $\mathrm{Y}$ & $\mathrm{Y}$ & $Y$ & $\mathrm{Y}$ & $\mathrm{Y}$ & & $\mathrm{Y}$ & $Y$ & $\mathrm{Y}$ & $\mathrm{Y}$ & 20 \\
\hline A4109 & $Y$ & $\mathrm{Y}$ & & $\mathrm{Y}$ & $\mathrm{Y}$ & $\mathrm{Y}$ & $Y$ & $\mathrm{Y}$ & $\mathrm{Y}$ & $Y$ & $\mathrm{Y}$ & $Y$ & & $\mathrm{Y}$ & & & & $\mathrm{Y}$ & $\mathrm{Y}$ & & $Y$ & & $\mathrm{Y}$ & $\mathrm{Y}$ & 17 \\
\hline A4207 & $Y$ & & & $\mathrm{Y}$ & $\mathrm{Y}$ & & & & $\mathrm{Y}$ & $Y$ & & & & $\mathrm{Y}$ & $\mathrm{Y}$ & & & $\mathrm{Y}$ & $Y$ & & & $Y$ & $\mathrm{Y}$ & $\mathrm{Y}$ & 12 \\
\hline A4214 & $Y$ & $\mathrm{Y}$ & & $\mathrm{Y}$ & $\mathrm{Y}$ & & $\mathrm{Y}$ & & $\mathrm{Y}$ & $Y$ & & & & $\mathrm{Y}$ & $\mathrm{Y}$ & $\mathrm{Y}$ & & $\mathrm{Y}$ & $\mathrm{Y}$ & $\mathrm{Y}$ & & & & & 13 \\
\hline A4208 & $\mathrm{Y}$ & $\mathrm{Y}$ & $\mathrm{Y}$ & $\mathrm{Y}$ & $\mathrm{Y}$ & & $Y$ & & & & & & & $Y$ & $\mathrm{Y}$ & & & & $\mathrm{Y}$ & & $\mathrm{Y}$ & & $\mathrm{Y}$ & & 11 \\
\hline A4210 & $Y$ & $\mathrm{Y}$ & $\mathrm{Y}$ & $Y$ & $\mathrm{Y}$ & $Y$ & $Y$ & $Y$ & $Y$ & $Y$ & $\mathrm{Y}$ & $Y$ & $\mathrm{Y}$ & $Y$ & $\mathrm{Y}$ & $\mathrm{Y}$ & $Y$ & $\mathrm{Y}$ & $Y$ & $\mathrm{Y}$ & $\mathrm{Y}$ & $\mathrm{Y}$ & $\mathrm{Y}$ & $Y$ & 24 \\
\hline A4221 & $Y$ & & & $\mathrm{Y}$ & $\mathrm{Y}$ & $\mathrm{Y}$ & & $\mathrm{Y}$ & & & & & & $Y$ & & $\mathrm{Y}$ & $Y$ & $\mathrm{Y}$ & & & $\mathrm{Y}$ & & $\mathrm{Y}$ & & 11 \\
\hline A5119 & $Y$ & $\mathrm{Y}$ & & $\mathrm{Y}$ & $\mathrm{Y}$ & $Y$ & & & & & & & & & $\mathrm{Y}$ & $\mathrm{Y}$ & & $Y$ & $\mathrm{Y}$ & & & $\mathrm{Y}$ & & & 10 \\
\hline A5118 & $\mathrm{Y}$ & $\mathrm{Y}$ & $Y$ & $\mathrm{Y}$ & & $\mathrm{Y}$ & $Y$ & $\mathrm{Y}$ & $\mathrm{Y}$ & & $\mathrm{Y}$ & $Y$ & & $Y$ & & $\mathrm{Y}$ & & $\mathrm{Y}$ & $Y$ & $\mathrm{Y}$ & $\mathrm{Y}$ & $\mathrm{Y}$ & $\mathrm{Y}$ & $\mathrm{Y}$ & 19 \\
\hline A5120 & $Y$ & $\mathrm{Y}$ & & $\mathrm{Y}$ & $\mathrm{Y}$ & $\mathrm{Y}$ & & $Y$ & $\mathrm{Y}$ & $Y$ & $\mathrm{Y}$ & $Y$ & $\mathrm{Y}$ & $\mathrm{Y}$ & $\mathrm{Y}$ & $\mathrm{Y}$ & $Y$ & $Y$ & $\mathrm{Y}$ & $\mathrm{Y}$ & $\mathrm{Y}$ & $\mathrm{Y}$ & $\mathrm{Y}$ & $\mathrm{Y}$ & 22 \\
\hline A5203 & $Y$ & $\mathrm{Y}$ & & $\mathrm{Y}$ & & & & $\mathrm{Y}$ & & $Y$ & & & & & & $\mathrm{Y}$ & & $\mathrm{Y}$ & & & & & $\mathrm{Y}$ & $\mathrm{Y}$ & 9 \\
\hline A5210 & $Y$ & & & $\mathrm{Y}$ & $\mathrm{Y}$ & $\mathrm{Y}$ & $\mathrm{Y}$ & $\mathrm{Y}$ & $\mathrm{Y}$ & $Y$ & & $Y$ & $\mathrm{Y}$ & $\mathrm{Y}$ & $\mathrm{Y}$ & $\mathrm{Y}$ & $Y$ & $\mathrm{Y}$ & $\mathrm{Y}$ & & $\mathrm{Y}$ & & $\mathrm{Y}$ & $\mathrm{Y}$ & 19 \\
\hline A5207 & $\mathrm{Y}$ & & $\mathrm{Y}$ & $\mathrm{Y}$ & $\mathrm{Y}$ & & & $Y$ & $\mathrm{Y}$ & Y & & & $\mathrm{Y}$ & $\mathrm{Y}$ & $\mathrm{Y}$ & & & $Y$ & $\mathrm{Y}$ & $Y$ & & $\mathrm{Y}$ & $\mathrm{Y}$ & $\mathrm{Y}$ & 16 \\
\hline
\end{tabular}

Fonte: dados da pesquisa

Habilidade acima da média Criatividade
Envolvimento com a tarefa

Ao analisar a tabela, pode-se perceber que todos os pais selecionaram os indicadores números um (aprende fácil e rapidamente) e doze (entende a importância da 
natureza) do anel Habilidade Acima da Média. O indicador dezenove (mostra insight e percepções incomuns) do anel Criatividade foi o menos selecionado pelos pais. Os pais dos alunos A3101 e A3202 selecionaram o menor número de indicadores em seus filhos, ambos com oito cada. Apenas os pais do aluno A4210 selecionaram todos os indicadores. $\mathrm{O}$ anel Criatividade foi o menos selecionado pelos pais.

Os pais, de forma geral, têm uma percepção acentuada em relação ao potencial de seus filhos. Fleith (2007) aponta a alta expectativa e também receio de identificações errôneas presentes nos pais das crianças. Neste estudo, percebeu-se que, mesmo com a alta valorização ao selecionar os itens, os pais demonstraram ter receio nas respostas escritas. Na ficha de caracterização de pais pudemos encontrar falas como "[...] não me julgo capaz para fazer esta análise, pois iria agir como pai e todo pai é babão, ou seja, sempre acha o filho muito inteligente".

Todavia, os professores foram mais moderados em suas respostas, quem sabe, pelo fato de terem um conhecimento superficial e até mesmo errôneo sobre o tema, esperando comportamentos excepcionais de seus alunos (Maia-Pinto e Fleith, 2002; Azevedo, 2008; Marques, 2010) conforme demonstrado na Tabela 2.

Tabela 2: Percepção de professores quanto a presença de indicadores de Superdotação em seus alunos

\begin{tabular}{|c|c|c|c|c|c|c|c|c|c|c|c|c|c|c|c|c|c|c|c|c|c|c|c|c|c|}
\hline \multirow{2}{*}{\begin{tabular}{|c|} 
Código do aluno \\
A3101
\end{tabular}} & \multicolumn{9}{|c|}{ Q01 Q06 Q07 Q12 Q13 Q14 Q16 Q21 Q23 } & \multicolumn{7}{|c|}{ Q17 Q19 Q22 Q04 Q02 Q10 Q11 } & \multicolumn{8}{|c|}{ Q18 Q20 Q24 Q15 Q03 Q05 Q08 Q09 } & \multirow{2}{*}{\begin{tabular}{|c|} 
TOTAL \\
8 \\
\end{tabular}} \\
\hline & & & $\mathrm{x}$ & & & $\mathrm{x}$ & $\mathrm{x}$ & & & $\mathrm{x}$ & $\mathrm{x}$ & & & & & $\mathrm{x}$ & $\mathrm{x}$ & & & & $\mathrm{x}$ & & & & \\
\hline A3106 & & & & $\mathrm{x}$ & & $\mathrm{x}$ & $\mathrm{x}$ & & $x$ & $\mathrm{x}$ & & & & & & $\mathrm{x}$ & & & $\mathrm{x}$ & & & & & & 7 \\
\hline A3105 & & $\mathrm{x}$ & & & $\mathrm{x}$ & & & $\mathrm{x}$ & $\mathrm{x}$ & & $\mathrm{x}$ & & $\mathrm{x}$ & $\mathrm{x}$ & & & $\mathrm{x}$ & & & & & & $\mathrm{x}$ & & 9 \\
\hline A3222 & $\mathrm{x}$ & & $\mathrm{x}$ & & $\mathrm{x}$ & $\mathrm{x}$ & $\mathrm{x}$ & & $x$ & & $\mathrm{x}$ & & $\mathrm{x}$ & $\mathrm{x}$ & $\mathrm{x}$ & $\mathrm{x}$ & $x$ & & & $x$ & $x$ & $\mathrm{x}$ & $\mathrm{x}$ & $x$ & 17 \\
\hline A3230 & & & & $x$ & & & $x$ & & & & & & & & & & & & & & $x$ & & $x$ & & 4 \\
\hline A3202 & $x$ & & & & & & & $x$ & & $x$ & & & $x$ & & $x$ & & $x$ & & $x$ & $x$ & & $x$ & & & 9 \\
\hline A4101 & & & & & & & & & & & & & & & & & & & & & & & & $x$ & 1 \\
\hline A4103 & & $x$ & $x$ & $x$ & & & & & & $x$ & $x$ & & $x$ & & $x$ & $x$ & $x$ & & & $x$ & $x$ & $x$ & & & 12 \\
\hline A4109 & $x$ & $x$ & $x$ & & $\mathrm{x}$ & $\mathrm{x}$ & $\mathrm{x}$ & $\mathrm{x}$ & $x$ & & $x$ & & $x$ & & $x$ & & $x$ & $\mathrm{x}$ & & $\mathrm{x}$ & & & & & 14 \\
\hline A4207 & & & $x$ & & $x$ & $\mathrm{x}$ & & $x$ & & & & $x$ & & & & & & & & & $x$ & & & $x$ & 7 \\
\hline A4214 & & & & $x$ & $\mathrm{x}$ & & & & & & & $x$ & $\mathrm{x}$ & & $x$ & & & & $x$ & & $x$ & & & $x$ & 8 \\
\hline A4208 & & & $x$ & & & & $\mathrm{X}$ & & $x$ & & $x$ & & $x$ & $x$ & & & $x$ & & & & & & $x$ & & 8 \\
\hline A4210 & & $x$ & & & $\mathrm{x}$ & $\mathrm{X}$ & & & & & & & & $\mathrm{x}$ & & $x$ & & & & & $x$ & & & & 6 \\
\hline A4221 & & $x$ & & & & & & $x$ & $x$ & $x$ & $x$ & & & & & & & & & & & & $x$ & & 6 \\
\hline A5119 & & $x$ & & & $\mathrm{x}$ & $\mathrm{x}$ & & $x$ & $\mathrm{x}$ & $x$ & $x$ & $x$ & $\mathrm{x}$ & & $x$ & & & $\mathrm{x}$ & $x$ & $x$ & & $x$ & $x$ & $x$ & 16 \\
\hline A5118 & & $x$ & & & $\mathrm{x}$ & $\mathrm{X}$ & & $\mathrm{x}$ & $x$ & $x$ & $\mathrm{x}$ & $x$ & & & $x$ & & & $\mathrm{x}$ & $\mathrm{x}$ & $\mathrm{x}$ & & $x$ & $x$ & $x$ & 15 \\
\hline A5120 & & $\mathrm{x}$ & $x$ & $x$ & $x$ & & $\mathrm{x}$ & & $x$ & & & & & $\mathrm{x}$ & & & $x$ & $\mathrm{x}$ & & & $x$ & & & $x$ & 11 \\
\hline A5203 & & $x$ & $x$ & $x$ & & $\mathrm{X}$ & & $\mathrm{X}$ & $\mathrm{x}$ & & & & $\mathrm{X}$ & $\mathrm{x}$ & & $x$ & $x$ & & & & & & $x$ & & 11 \\
\hline A5210 & $x$ & $x$ & & & $\mathrm{x}$ & $\mathrm{x}$ & $\mathrm{x}$ & & & & $\mathrm{x}$ & & $x$ & $\mathrm{x}$ & $x$ & & $x$ & & & $x$ & $x$ & $x$ & $x$ & & 14 \\
\hline A5207 & $x$ & $x$ & $x$ & & & $x$ & $\mathrm{x}$ & & & & $x$ & & & $\mathrm{x}$ & $x$ & & $x$ & $\mathrm{x}$ & & $\mathrm{x}$ & & $x$ & & & 12 \\
\hline
\end{tabular}

Fonte: dados da pesquisa

Habilidade acima da média Criatividade
Envolvimento com a tarefa

Através da tabela, pode-se perceber que o aluno A4101 foi apontado pelo professor como tendo apenas um indicador. Já o aluno A3222 foi o que, de acordo com 
o ponto de vista do professor apresenta mais indicadores, totalizando dezessete. $\mathrm{O}$ indicador menos selecionado por professores foi o indicador vinte e dois (resiste à rotina e repetição) do anel Criatividade, com apenas quatro marcações. Os indicadores mais marcados pelos professores foram o seis, quatorze, dezenove e dezoito, com onze marcações cada.

O número reduzido de indicadores percebidos pelos professores, pode ser devido a um conhecimento superficial do conceito ou a falta de orientação específica, corroborando com Maia-Pinto e Fleith (2002).

Ao cruzar os dados dos pais e professores, percebeu-se que em cada sala, dois ou três casos se destacaram simultaneamente sob o olhar dos professores e pais, apresentando indicadores apontados pelos dois grupos em comum. Tais congruências, podem ser encontradas na Tabela 3, onde o Y representa a percepção de pais e o X, de professores

Tabela 3: Percepção em comum de professores (X) e pais (Y) quanto a presença de indicadores de Superdotação

\begin{tabular}{|c|c|c|c|c|c|c|c|c|c|c|c|c|c|c|c|c|c|c|c|c|c|c|c|c|c|}
\hline Código do aluno & Q01 & Q06 & Q07 & Q12 & Q13 & Q14 & Q16 & Q21 & Q23 & Q17 & Q19 & $\mathrm{Q} 22$ & Q04 & $\mathrm{Q} 02$ & Q10 & Q11 & Q18 & $\bar{Q} 20$ & $\overline{Q 24}$ & Q15 & $\overline{\mathrm{Q} 03}$ & $\mathrm{Q} 05$ & Q08 & Q09 & TOTAL \\
\hline A3101 & $\mathbf{Y}$ & $\mathbf{Y}$ & YX & $\mathrm{Y}$ & $\mathrm{Y}$ & $\mathrm{x}$ & $\mathrm{x}$ & & & $\mathrm{x}$ & $\mathrm{x}$ & & & & $\mathrm{Y}$ & $\mathrm{x}$ & $\mathrm{x}$ & & $\mathbf{Y}$ & & $\mathrm{x}$ & & & $\mathbf{Y}$ & 1 \\
\hline A3106 & $\mathbf{Y}$ & $\mathbf{Y}$ & & $Y X$ & & YX & YX & $\mathbf{Y}$ & YX & YX & & $\mathbf{Y}$ & & & $\mathrm{Y}$ & $\mathrm{YX}$ & & $\mathbf{Y}$ & YX & $\mathbf{Y}$ & & & & & 7 \\
\hline A3105 & $\mathbf{Y}$ & YX & & $\mathbf{Y}$ & $Y X$ & $\mathbf{Y}$ & $\mathbf{Y}$ & YX & YX & $\mathbf{Y}$ & YX & $\mathbf{Y}$ & YX & YX & $\mathrm{Y}$ & & YX & $\mathbf{Y}$ & $\mathbf{Y}$ & $\mathbf{Y}$ & & & YX & $\mathbf{Y}$ & 9 \\
\hline A3222 & $Y X$ & & $Y X$ & $\begin{array}{l}\mathrm{Y} \\
\mathrm{y}\end{array}$ & $Y X$ & $Y X$ & $x$ & & $\mathrm{YX}$ & $\mathbf{Y}$ & $x$ & & $Y X$ & $x$ & $\mathrm{x}$ & $\mathrm{YX}$ & $x$ & & $\mathbf{Y}$ & $\mathbf{Y X}$ & $\mathrm{YX}$ & $Y X$ & YX & $Y X$ & 12 \\
\hline A3230 & $\mathbf{Y}$ & $\mathbf{Y}$ & $\mathrm{Y}$ & $Y X$ & $\mathbf{Y}$ & & $Y X$ & $\mathbf{Y}$ & $\mathbf{Y}$ & & $\mathbf{Y}$ & $\mathbf{Y}$ & $\mathbf{Y}$ & $\mathbf{Y}$ & & & $\mathbf{Y}$ & & $\mathbf{Y}$ & & YX & $\mathbf{Y}$ & YX & $\mathbf{Y}$ & 4 \\
\hline A3202 & $Y X$ & & $\mathbf{Y}$ & $\mathrm{Y}$ & & & $\mathbf{Y}$ & $x$ & & $\mathrm{x}$ & & & $x$ & $\mathbf{Y}$ & $Y X$ & & YX & & $\mathrm{x}$ & $\mathrm{x}$ & $\mathbf{Y}$ & $x$ & & & 3 \\
\hline A4101 & $\mathrm{Y}$ & $\mathrm{Y}$ & & $\begin{array}{l}\mathrm{Y} \\
\mathrm{y}\end{array}$ & & & $\mathbf{Y}$ & & $\mathbf{Y}$ & $\mathrm{Y}$ & & & & $\mathbf{Y}$ & $\begin{array}{l}\mathbf{Y} \\
\end{array}$ & $\mathbf{Y}$ & & $\mathbf{Y}$ & $\mathbf{Y}$ & & & & $\mathbf{Y}$ & $\mathrm{YX}$ & 1 \\
\hline A4103 & $\mathbf{Y}$ & $Y X$ & $x$ & $Y X$ & $Y$ & $\mathrm{Y}$ & $\mathbf{Y}$ & $\mathbf{Y}$ & $\mathbf{Y}$ & $Y X$ & $x$ & $\mathbf{Y}$ & $x$ & $\mathbf{Y}$ & $Y X$ & $\mathrm{YX}$ & $Y X$ & $\mathbf{Y}$ & $\mathbf{Y}$ & $x$ & YX & $Y X$ & $Y$ & $\mathbf{Y}$ & 8 \\
\hline A4109 & $Y X$ & $Y X$ & $x$ & $\mathbf{Y}$ & $Y X$ & $Y X$ & $Y X$ & $Y X$ & $\mathbf{Y X}$ & $Y$ & $Y X$ & $\mathbf{Y}$ & $x$ & $\mathbf{Y}$ & $x$ & & $x$ & $\mathbf{Y X}$ & $Y$ & $\mathrm{X}$ & $\mathbf{Y}$ & & $Y$ & $Y$ & 9 \\
\hline A4207 & $\mathbf{Y}$ & & $x$ & $Y$ & $Y X$ & $x$ & & $x$ & $Y$ & $Y$ & & $x$ & & $Y$ & $Y$ & & & $Y$ & $Y$ & & $x$ & $\mathbf{Y}$ & $Y$ & $Y X$ & 2 \\
\hline 21 & $\mathbf{Y}$ & $\mathbf{Y}$ & & $Y X$ & $Y X$ & & $\mathbf{Y}$ & & $\mathbf{Y}$ & $Y$ & & $x$ & $\mathrm{x}$ & $\mathbf{Y}$ & $Y X$ & $\mathbf{Y}$ & & $\mathbf{Y}$ & YX & $\mathbf{Y}$ & $\mathrm{x}$ & & & $x$ & 4 \\
\hline A & $\mathbf{Y}$ & $Y$ & $Y X$ & $\mathrm{Y}$ & $\mathbf{Y}$ & & YX & & $x$ & & $x$ & & $x$ & $Y X$ & $\mathrm{Y}$ & & $x$ & & $\mathbf{Y}$ & & $\mathbf{Y}$ & & $Y X$ & & 4 \\
\hline A & $\mathbf{Y}$ & $Y X$ & $\mathbf{Y}$ & $\mathbf{Y}$ & $Y X$ & $Y X$ & $\mathbf{Y}$ & $\mathbf{Y}$ & $\mathbf{Y}$ & $\mathbf{Y}$ & $\mathbf{Y}$ & $Y$ & $\mathbf{Y}$ & $Y X$ & $\mathbf{Y}$ & $\mathrm{YX}$ & $\mathbf{Y}$ & $\mathbf{Y}$ & $\mathbf{Y}$ & $\mathbf{Y}$ & $\mathbf{Y X}$ & $\mathbf{Y}$ & $Y$ & $\mathbf{Y}$ & 6 \\
\hline A & $\mathbf{Y}$ & $x$ & & $\mathbf{Y}$ & $\mathbf{Y}$ & $\mathrm{Y}$ & & $Y X$ & $x$ & $x$ & $x$ & & & $\mathbf{Y}$ & & $Y$ & $\mathbf{Y}$ & $\mathbf{Y}$ & & & $\mathbf{Y}$ & & $Y X$ & & 2 \\
\hline A5119 & $Y$ & $Y X$ & & $\mathrm{Y}$ & $Y X$ & $Y X$ & & $x$ & $x$ & $x$ & $x$ & $x$ & $x$ & & $Y X$ & $Y$ & & YX & YX & $x$ & & $Y X$ & $x$ & $x$ & 7 \\
\hline A5118 & $\mathrm{Y}$ & $Y X$ & $Y$ & $\mathrm{Y}$ & $x$ & $Y X$ & $Y$ & YX & YX & $x$ & YX & $Y X$ & & $Y$ & $x$ & $Y$ & & YX & YX & YX & $Y$ & $Y X$ & $Y X$ & $Y X$ & 12 \\
\hline A5120 & $\mathrm{Y}$ & $Y X$ & $x$ & $Y X$ & $Y X$ & $Y$ & $x$ & $\mathrm{Y}$ & YX & $\mathrm{Y}$ & $\mathrm{Y}$ & $Y$ & $Y$ & $Y X$ & $\mathrm{Y}$ & $Y$ & YX & YX & $\mathrm{Y}$ & $Y$ & $Y X$ & $Y$ & $Y$ & $Y X$ & 9 \\
\hline A5203 & $Y$ & $Y X$ & $x$ & $Y X$ & & $x$ & & YX & $x$ & $\mathrm{Y}$ & & & $x$ & $x$ & & $\begin{array}{ll}x X \\
\end{array}$ & $x$ & $Y$ & & & & & $\mathrm{YX}$ & $\mathrm{Y}$ & 5 \\
\hline A5210 & $Y X$ & $x$ & & $\mathrm{Y}$ & $Y X$ & $Y X$ & YX & $\mathbf{Y}$ & $Y$ & $Y$ & $\mathrm{x}$ & $Y$ & $\begin{array}{l}X X \\
\end{array}$ & $\begin{array}{l}Y X \\
\end{array}$ & $Y X$ & $\mathbf{Y}$ & YX & $Y$ & I & 凡 & YX & $x$ & $Y X$ & $\mathbf{Y}$ & 10 \\
\hline A5207 & YX & $x$ & $Y X$ & $\mathrm{Y}$ & $\mathrm{Y}$ & $x$ & $x$ & $Y$ & $Y$ & $Y$ & $x$ & & $Y$ & $Y X$ & $Y X$ & & $x$ & YX & $Y$ & YX & & $Y X$ & $Y$ & $\mathrm{Y}$ & 7 \\
\hline
\end{tabular}

Fonte: dados da pesquisa

Habilidade acima da média Criatividade
Envolvimento com a tarefa

A Tabela 3 permite comparar a percepção dos pais (Y) e professores (X) da presença de indicadores de Superdotação/Altas Habilidades em cada aluno. Dos vinte alunos destacados, podemos ver que os alunos A3222 e A5118 apresentam o maior número de percepções em comum, totalizando doze. O segundo aluno com um grande número de congruências, somando dez indicadores em comum, foi o A5210. Os alunos A3105, A4109 e A5120, apresentam nove indicadores em paridade, o A4103 apresenta 
oito, os alunos A3106, A5119 e A5207 apresentam sete indicadores e o A4210 apresentou seis indicadores em comum. Os demais apresentaram cinco indicadores ou menos em paridade.

Nota-se que as perguntas um e doze, referentes ao anel de Habilidade Acima da Média, foram apontadas por todos os pais, e a pergunta dezenove, referente à Criatividade, foi a com menor número de sinalização dos pais, apenas com seis marcações.

Os professores, contudo, foram mais cuidadosos em suas respostas. Os indicadores mais percebidos pelos docentes foram os números: seis em Habilidade Acima da Média, dezenove em Criatividade e dezoito em Envolvimento com a Tarefa, totalizando onze alunos em cada. O indicador vinte e dois foi notado em apenas quatro crianças, sendo referente à Criatividade e representando o menor número de indicações.

A média de indicações dos pais foi de cerca de quinze questões, enquanto a dos professores foi de apenas nove, demonstrando o maior cuidado e/ou receio por parte dos docentes.

Dos 160 questionários preenchidos por pais e professores, 20 alunos se destacaram com mais indicadores em relação aos colegas, ou seja, 12\%. Dos 20 alunos destacados, $75 \%$ apresentaram indicadores nos três anéis, $15 \%$ em dois anéis e $10 \%$ em apenas um.

Em ambos os pontos de vista, o anel de Habilidade Acima da Média foi percebido com mais facilidade. $\mathrm{O}$ anel de Criatividade teve o menor número de percepções em ambos os participantes, podendo ser devido ao não recebimento de incentivo de ambos. Marques (2010) afirma que a criança que tem criatividade é bemhumorada, independente e pode ter dificuldades acadêmicas. Esse fato, pode levar os professores a enxerga-lo como mau aluno e não levar em consideração a potencialidade dentro dos indicadores. Na coleta de dados, a afirmação de uma participante mãe se destacou: "Observo que a escolarização de certa maneira "doutrinou" seu pensamento criativo, que antes, era bem mais livre".

\section{Considerações finais}

No andamento da pesquisa percebeu-se a disparidade entre a percepção de pais e professores em relação a S/AH. Corroborando com o ponto de vista de Fleith (2007), os pais demonstraram grandes expectativas quanto ao potencial de seus filhos. Já os 
professores se sentiram menos seguros ao identificarem o potencial de seus alunos, de acordo com Maia-Pinto e Fleith, 2002.

Dentro da Teoria dos três anéis de Renzulli (1986), chamou a atenção o baixo número de identificação dos indicadores de Criatividade, que, segundo o autor, envolve originalidade de pensamento e talento para solucionar problemas. Tais aspectos são muito importantes para o desenvolvimento tanto da sociedade, que precisa de indivíduos talentosos e criativos, quanto individualmente.

Pais e professores também apresentaram conhecimento superficial do tema e receio de identificações errôneas, fazendo necessário maiores subsídios para compreensão da S/AH, como apontado por Marques (2010).

\section{REFERÊNCIAS}

AZEVEDO, T. M. P. A percepção dos educadores sobre as altas habilidades e a possibilidade de atendimento aos alunos superdotados no ensino regular. Santa Maria, RS. 2008.

BRASIL. MEC. CNE/CEB. Resolução n. 2 de 11 de setembro de 2001. Disponível em: <http://portal.mec.gov.br/cne/arquivos/pdf/CEB0201.pdf>. Acesso em: 07 mar. 2017.

BRASIL. MEC. Política Nacional de Educação Especial na Perspectiva da Educação Inclusiva. Brasília, DF: MEC/ PNEEPEI, 2008.

BRASIL. MEC. Saberes e práticas da inclusão: Desenvolvendo competências para o atendimento ás necessidades educacionais especiais de alunos com altas habilidades/superdotação. 2a ed Brasília: MEC Secretaria de Educação Especial, 2006.

CAMPBELL, J. R. e VERNA, M.A. Messages from the field: American teachers of the gifted talk back to the research community. San Diego, CA, 1998.

FLEITH D. S. A construção de práticas educacionais para alunos com altas habilidades/superdotação: o aluno e a família. vol. 3, Brasília: Ministério da Educação, Secretaria de Educação Especial, 2007.

FONSECA, J. J. S. Metodologia da pesquisa científica. Fortaleza: UEC, 2002. Apostila.

GAGNÉ, F. Building gifts into talents: detailed overview of the DMGT 2.0. Université du Québec a Montreal Department of psychology, 2009.

GAGNÉ, F. A Differentiated Model of Gifitedness and Talent. Université du Québec a Montreal Department of psychology, 2000.

GARDNER, H. Estruturas da mente. Porto Alegre: Artes Médicas, 1994. 
GUENTHER, Z. C. Desenvolver capacidade e talentos: um conceito de inclusão. Petrópolis: Vozes, 2006.

HOCHMAN B.; NAHAS F. X.; OLIVEIRA FILHO R. S.; FERREIRA, L. M. Desenhos de pesquisa. Acta Cir Bras [serial online] 2005;20 Suppl. 2:02-9.

KAHL, J. A concepção do professor de PROEJA quanto às altas habilidades/superdotação. 2011. 65 f. Dissertação (Mestrado) - Curso de Educação, Universidade Federal de Santa Maria, Santa Maria, 2011.

LOPES, B. J. S.; GIL, M. E. C. A. Altas habilidades/superdotação percebidas pelas mães nos seus filhos com deficiência visual. v. 22, n. 2, p. 203-220, abr. 2016.

MAIA-PINTO, R. R.; FLEITH, D. S. Percepção de professores sobre alunos superdotados. Estudos de Psicologia, Campinas, v. 19, n. 1, p. 78-90, abr. 2002.

MARQUES, C. R. Levantamento de indicadores de crianças com altas habilidades e superdotação em Juquiticabal/São Paulo. 2010. 167f. Dissertação (Mestrado em Educação Especial) - Universidade Federal de São Carlos, São Carlos, 2010.

OLIVEIRA, P. S. Investigando e analisando as percepções de pais acerca de um filho com indicadores de altas habilidades/superdotação. 2008. 32 f. (Mestrado em Educação Especial) - Universidade Federal de Santa Maria. Santa Maria, 2008.

RECH, A. D. Estudo de uma criança com características de Altas Habilidades: Problematizando questões referentes a inclusão escolar. Dissertação (Mestrado em Educação Especial) - Universidade Federal de Santa Maria. Santa Maria, 2007.

RENZULLI, J. S. The Three-ring conception of giftedness: A developmental model for creative productivity. New York: Cambridge University Press, 1986.

SANTOS. L. C.; PERIPOLLI. A. Altas Habilidades/Superdotação: clarificando concepções e (re)significando ideias imagéticas do senso comum. Revista do Difere, v. 1, n. 2 , dez/2011.

SILVA, P. V. C.; FLEITH, D. S. A influência da família no desenvolvimento da superdotação. Psicologia escolar e educacional, dez. 2008.

VIRGOLIM, A. M. R. Altas habilidades/superdotação: encorajando potenciais. Brasília: Ministério da Educação, Secretaria de Educação Especial, 2007. 


\section{Como referenciar este artigo}

MEDEIROS, Alexandra Imaculada de Oliveira et al. Superdotação/altas habilidades percebidas por pais e professores do ensino fundamental, nível I. Revista on line de Política e Gestão Educacional, Araraquara, v. 21, n. esp.2, p. 1179-1194, nov. 2017. Disponível em: <http://dx.doi.org/10.22633/rpge.v21.n.esp2.2017.10432>. EISSN:1519-9029.

Submetido em: 25/09/2017

Aprovado em: 30/10/2017 